\title{
РОМАНСКИЕ ЯЗЫКИ
}

УДК $811.133 .1: 81^{`} 36$

DOI: $10.18384 / 2310-712 \mathrm{X}-2021-5-36-45$

\section{ФУНКЦИОНИРОВАНИЕ ОБЩЕВОПРОСИТЕЛЬНЫХ ПРЕДЛОЖЕНИЙ С ПРЯМЫМ ПОРЯДКОМ СЛОВ ВО ФРАНЦУЗСКОМ ЯЗЫКЕ (НА МАТЕРИАЛЕ ХУДОЖЕСТВЕННЫХ ТЕКСТОВ XI-XVII ВЕКОВ)}

\author{
Зубкова М. Н.', Дроздова Ю. А. ${ }^{2}$ \\ ${ }^{1}$ Московский государственный институт международных отношений (университет) \\ Министерства иностранных дел Российской Федерации \\ 119454, г. Москва, пр-т Вернадского, д. 76, Российская Федерация \\ ${ }^{2}$ Всероссийская академия внешней торговли Министерства экономического развития \\ Российской Федерации \\ 1192856, г. Москва, Воробьевское ш., д. 6А, Российская Федерация
}

\begin{abstract}
Аннотация
Целью написания настоящей статьи выступает выявление основных характеристик общевопросительных предложений с прямым порядком слов во фрранцузском языке в аспекте диахронии. Рассматривается эволюция семантико-грамматических особенностей вопроса SVтипа в период с XI по XVII вв. Анализируются отличия вопросительной конструкции с прямым порядком слов от вопроса с инверсией с точки зрения семантики и синтаксиса.

Процедура и методы. Авторами проанализирован значительный корпус примеров из текстов художественной литературы XI-XVII вв., полученных методом сплошной выборки. Методологической базой исследования являлись: сравнительно-сопоставительный метод, метод лингвистического наблюдения и описания, приёмы контекстного, коммуникативно-прагматического и контрастивного анализа изучаемых явлений в синхронии и диахронии.

Результаты. В процессе исследования были выявлены основные отличия вопроса с прямым порядком слов от вопроса с инверсией, с одной стороны, и от повествовательного предложения, с другой стороны. Было доказано, что предпочтение структуры SV объясняется рядом взаимообусловленных фракторов как структурно-семантического, так и прагматико-социолингвистического характера.
\end{abstract}

Теоретическая и/или практическая значимость. Полученные результаты исследования могут быть использованы при преподавании курса истории фрранцузского языка, в спецкурсах по теоретической грамматике на уровне старших курсов бакалавриата, а также в магистратуре.

Ключевые слова: общевопросительное предложение, прямой порядок слов, вопросительная целеустановка, запрос подтверждения информации, вопросительная конструкция, инверсия

(с) СС ВҮ Зубкова М. Н., Дроздова Ю. А., 2021. 


\title{
FUNCTIONS OF GENERAL QUESTIONS WITH DIRECT WORD ORDER IN FRENCH (BASED ON LITERARY TEXTS OF THE $11^{\text {TH }}-17^{\text {TH }}$ CENTURIES)
}

\author{
M. Zubkova' ${ }^{1}$ Yu. Drozdova² \\ ${ }^{1}$ Moscow State Institute of International Relations (University) \\ of the Ministry of Foreign Affairs of the Russian Federation \\ 76 prospekt Vernadskogo, Moscow 119454, Russian Federation \\ ${ }^{2}$ Russian Foreign Trade Academy \\ 6A Vorob'evskoe shosse, Moscow 1192856, Russian Federation
}

\section{Abstract}

Aim. The following article presents the analysis of main characteristics and distinctive features of interrogative sentences with direct word order in French language in diachronic aspect. Evolution of semantic and grammar particularities of SV-type question in XI-XVII centuries is considered. Semantic and syntactic differences of interrogative construction with direct word order and interrogative with inversion are analyzed.

Methodology. An important corpus of examples from belles-lettres of XI-XVII centuries chosen by continuous sampling method was analyzed. The methodological basis of the research are: contrastive-comparative method, linguistic notice and description method, context, communicative-pragmatic and contrastive analysis of studied facts in synchronic and diachronic aspects.

Results. The analysis detected main differences of interrogative with direct word order from interrogative with inversion to the one hand and from narrative sentence to the other hand. The research proved that the predilection of SV structure is explained by some interdependent structural-semantic and pragmatic-sociolinguistic factors.

Research implications. The research results can be used in teaching of French language history and special courses of theoretical grammar at the bachelor and master levels.

Keywords: general interrogative, direct word order, interrogative object, request for confirmation, interrogative construction, inversion

\section{Введение}

Порядок слов относится к хорошо изученным явлениям французского синтаксиса. В работах как отечественных, так и зарубежных авторов не раз ставился вопрос о положении главных и второстепенных членов в предложении, а также о факторах, влияющих на порядок слов $[1-6 ; 10 ; 12 ; 14 ; 17 ; 18]$.

В современном французском языке порядок слов - Sujet-Verbe-Complément является нормой для повествовательного предложения, однако возможное построение вопросительного предложения с прямым порядком слов приводит к тому, что границы между вопросительным и повествовательным предложениями размываются. С этой тенденцией связан ряд теоретических и практических проблем, которые требуют решения, причём не только в плане синхронии, но и в плане диахронии.

Целью исследования, послужившего основой данной статьи, выступают выявление основных семантических, синтаксических и прагматических особенностей общевопросительных предложений с прямым порядком слов во французском языке в диахронном аспекте, а также разграничение вопросительной конструкции типа SV и вопроса с инверсией или est-ce que, с одной стороны, и повествовательного предложения, с другой стороны.

Анализ был проведён на материале корпуса примеров из текстов XI-XVII вв., полученных методом сплошной выборки.

Методологическую базу исследования составили: сравнительно-сопоставитель- 
ный метод, метод лингвистического наблюдения и описания, приёмы контекстного, коммуникативно-прагматического и контрастивного анализа изучаемых явлений в синхронии и диахронии.

\section{Основные отличия вопроса типа SV от конструкций с инверсией или est-ce que и от повествовательного предложения}

Как показало проведённое исследование, общевопросительное предложение с прямым порядком слов характеризуется рядом отличительных черт с точки зрения семантики, прагматики и функциональной целеустановки, что позволяет отграничить вопрос SV-типа как от повествовательного предложения, так и от вопроса с инверсией или est-ce que.

Основное значение интеррогатива это направленность на поиск информации, т. е. на восполнение отсутствующих у говорящего сведений об объекте разговора. Лингвисты говорят об “interrogation dubitative”, где говорящий указывает собеседнику, что он не может в полной мере судить о правдивости предмета разговора [15]. Это значение присуще конструкциям с инверсией или оборотом est-ce que.

Вопрос с SV отличается от данных типов вопросов прежде всего по своей функции. Как отмечает Н. М. Васильева, у структуры с прямым порядком слов ослабляется вопросительная целеустановка: “... la valeur interrogative de la phrase s'affaiblit ou s'efface complètement et devient proche de l'assertion" [3, с. 27]. Значением SV оказывается в таком случае запрос подтверждения информации ("la demande de la confirmation"). В связи с этим, использование вопросительного предложения типа SV исключено в высказываниях со значением сомнения, когда собеседник хочет найти ответ на свой вопрос и сам не склоняется к какому-либо определённому варианту [15].

Интересен анализ, проведённый М.Б. М. Хансен, которая рассматривает функции инверсии, est-ce que и прямого порядка слов с точки зрения диалогического взаимодействия (interaction) на базе диалогов устной речи. Автор выделяет две прагматические функции вопросов: "1. The speaker is signaling to the hearer that it is relevant for someone (prototypically, but not necessarily the speaker herself) to wonder about the validity of the proposition expressed. 2. The speaker is appealing to the hearer for a reaction to that proposition" (1. Адресант сигнализирует адресату, что кто-либо (не обязательно сам адресант) интересуется истинностью того или иного предложения. 2. Адресант призывает адресата отреагировать каким-либо образом на это предложение.) [15, с. 467]. По мнению исследователя, вопрос SV выполняет вторую функцию, и он более типичен в общении между людьми, которые являются близкими друзьями или принадлежат к одному социальному классу.

Что же касается определённой близости структур общего вопроса с прямым порядком слов и повествовательного предложения, необходимо учитывать ряд факторов, позволяющих провести некоторую границу между ними.

Отличительным признаком вопросительного предложения может служить вопросительный знак на письме и восходящая интонация - в устной речи [13, p. 1227; 20, p. 391; 3, с. 24]. Однако важно подчеркнуть, что многие авторы ставят под сомнение релевантность фонетического критерия [8, р. 120-130; 21]. Экспериментальные исследования показывают, что мелодический рисунок высказывания зависит от многих факторов (“... l'intonation renseigne sur l'attitude du locuteur, sur ses connaissances, et même sur la dimension dialogique” («... интонация говорит об отношении адресанта, его знаниях и даже об особенностях диалогической речи») [7, p. 51]), поэтому восходящая интонация не является однозначным показателем общего вопроса.

Таким образом, отличия общего вопроса структуры SV от повествовательного предложения минимальны, поскольку 
ни пунктуационный, ни фонетический критерии не являются однозначными.

Учитывая ряд схожих признаков с повествовательным предложением, можно говорить о том, что вопрос SV занимает промежуточное положение между утверждением и собственно вопросом. Его основным значением, в отличие от инверсии и оборота est-ce que, является запрос подтверждения информации.

\section{Результаты диахронного исследования эволюции вопросительной конструкции SV-типа на основе анализа языкового материала XI-XVII вв.}

Базируясь на результатах многочисленных статистических исследований, можно утверждать, что в современном языке вопрос с прямым порядком слов является доминирующим типом $[6 ; 11$; $15 ; 19]$. Подобное явление объясняется логикой исторического развития языка. Об этом говорит Л. Фуле (L. Foulet): “... de 1350 à 1650 l'effort de la langue a consisté principalement à faire triompher l'ordre sujet-verbe-complément, en d'autres termes à se débarrasser tant bien que mal des nombreuses inversions dont elle avait hérité et qui étaient désormais contraires à son génie" («... в период с 1350 по 1650 гг. развитие языка привело к повсеместному распространению порядка слов 'подлежащее сказуемое - прямое дополнение' другими словами, язык избавился в определённой степени от многочисленных видов инверсий, которые он унаследовал и которые противоречили его сути» [9, р. 262]. Так, эволюция порядка слов во французском вопросительном предложении шла по пути от VSC к SVC в общем вопросе, и от схемы QVS к SVQ - в частном.

Наиболее ранней формой построения вопроса являлась, безусловно, инверсия, и вплоть до XV в. она оставалась наиболее типичным средством выражения значения вопросительности.

Как отмечают исследователи, прямой порядок слов появляется сначала в при- даточных предложениях (уже в XII в.), в том числе в косвенных вопросах. В простом вопросительном предложении подобная структура начинает широко использоваться с XVII в. [13, р. 1227; 16, p. 76]. Таким образом, придаточное предложение предваряет развитие независимых предложений в плане порядка слов.

Это положение подтверждается результатами анализа значительного корпуса фактического материала. В процессе исследования было выявлено, что конструкция с прямым порядком слов встречается в составе сложносочинённого вопросительного предложения, а именно, во второй его части, в то время как в первой части содержится инверсия. Данное явление зафиксировано в текстах разных периодов (с XII по XIV вв.).

A) Dunc, n'este-vos li plus vaillant,

E nos sumes li meins puissant?

N'est'os meuz nez, plus hauz de nos, Plus forz et plus chevaleros ? ${ }^{1}$ - XII в.

B) Suesfrent il tuit aussi si grant dolor, Ou il dient le mal q'il ont d'amor? ${ }^{2}-$ XIII в. C) Fait il le sourt, ou il est mournes? ? $^{3}$ XIV в.

В ходе анализа художественных текстов установлено, что прямой порядок слов в простых общевопросительных предложениях эпизодически встречается уже в XII в. В старофранцузском языке такое построение предложения было характерно для т. н. «вопросов-эхо» или восклицательных предложений.

"Je le te dirai," fait Merlins, "car je sai bien que tu me veuls demander." "Tu le sés?" fait li rois: "encore ne le t'ai je pas dit".

\footnotetext{
1 Benoît de Sainte-Maure. Chronique des ducs de Normandie. Vol. II / publ. d'après un manuscrit de musée britannique par Francisque Michel. Paris: Imprimerie Royale, 1838. P. 308.

2 Les chansons de Thibaut de Champagne, roi de Navarre / ed. critique, publiée par A. Wallensköld. Paris: Librarie Ancienne Édouard Champion, 1925. P. 153.

3 Miracles de Nostre-Dame, par personnages. T. 2 / publiés d'après le ms. de la Bibliothèque nationale, par G. Paris, U. Robert. Paris: Librairie de Firmin Didot et Cie, 1877. P. 44.

4 Robert de Boron. Merlin: roman en prose du XIIIe siècle. T. 2 / publ. avec la mise en prose du poème de Merlin de Robert de Boron d'après le manuscrit ap-
} 
B XIV-XV вв. сфера употребления прямого порядка слов в вопросе расширяется. Основными контекстами для данных структур в этот период являются следующие:

1. Структура SV используется в случае, если говорящий просит у собеседника подтверждения информации, содержащейся в вопросе. Это значение можно считать основным и наиболее распространённым.

- Et vous savez qu'elle est muette?

Ne parle point.

- Sire, ne me chaut de ce point,

Tout a un mot $^{1}$.

В этом случае характерны вставки наречий типа donc, alors, что усиливает значение подтверждения.

Celi dist : Tu vels donc morir?

- Osis seras, n’i pues faillir ${ }^{2}$.

Нагляден в этом плане диалог Христа с Пилатом, где последний просит, чтобы Тот признал свою вину.

- Tu es donc roy?

- Tu le dis,

et m'as le nom de roy donné.

2. SV используется для выражения дополнительных значений (возмущения, удивления, недоумения и т. д.).

A) Lors la dame respond et dit: Vraiment, mes chieres commeres et cousines, je ne scey que faire, et mien ne scey chevir, tant est mal homme et divers. Il est mal homme, dit l'une d'elles?

partenant à M. Alfred H. Huth par G. Paris, J. Ulrich. Paris: Librairie de Firmin Didot et Cie, 1886. P. 161.

1 Miracles de Nostre-Dame, par personnages. T. 6 / publiés d'après le ms. de la Bibliothèque nationale, par G. Paris, U. Robert. Paris: Librairie de Firmin Didot et Cie, 1881. P. 72.

2 Renaut de Beaujeu. Le bel inconnu ou Giglain fils de messire Gauvain et de la feé aux blanches mains: poème de la Table Ronde ([Fac-sim.]) / publ. d'après le manuscrit unique de Londres, avec une introd. et un glossaire par C. Hippeau. Paris: Chez Auguste Aubry, 1860. P. 26.

3 Le Mystère de la Passion, d'Arnoul Greban / publié d'après les manuscrits de Paris, avec une introduction et un glossaire. Paris: Gallimard, 1987. P. 281.

4 Les quinze joyes de mariage [attribueìes à A. de La Sale] / nouv. éd., avec les variantes des anciennes éditions,
B) - Pierre, il vous convient ensuivir:

baillez vos piez, si le serez.

- Ha! sire, et vous me laverez

les piez? est ce chose advenant?

- Ce que je te fais maintenant

tu ne sces; après le sçaras ${ }^{5}$.

C) - Ne vous souvient-il, fait-elle, comment je fus tant malade de noustre enfant, et que je me voué à Nostre- Dame du Puy ... ; et vous n'en faites compte? - Avoy, fait-il m'amie, ne savez-vous pas comment j'ay tant a fure que je ne scey auquel obéir ?6

Вопрос часто сопровождается междометиями comment, quoi, allons.

Comment donc, vous me laisserez,

enffans, pour suir ung estrange?

Следующий пример является яркой демонстрацией чёткого семантического различия между вопросительной конструкцией с прямым порядком слов и с инверсией: говорящий пытается угадать, какой подарок приготовил его собеседник, последовательно задавая ему вопросы. Примечательно, что здесь сочетаются два указанных типа вопросов. Можно предположить, что в случае с SV говорящий уверен в том, что он даёт правильную версию и что его собеседник подтвердит его догадку. В случае же с инверсией у него нет такой уверенности.

- J'ay bien advisé quel present

je luy feray, qui sera digne.

- Quel, je te pry?

- Or adevine,

et tu orras bonne sornette.

- Tu luy veulx donner ta houlette

ou ton beau chapelet troué?

- Encor ne l'as tu pas trouvé:

une notice bibliographique et des notes par P. Jannet. Paris: Chez P. Jannet Libraire, 1853. P. 29.

5 Le Mystère de la Passion, d'Arnoul Greban / publié d'après les manuscrits de Paris, avec une introduction et un glossaire. Paris: Gallimard, 1987. P. 233.

6 Les quinze joyes de mariage [attribueìes à A. de La Sale] / nouv. éd., avec les variantes des anciennes éditions, une notice bibliographique et des notes par P. Jannet. Paris: Chez P. Jannet Libraire, 1853. P. 98.

7 Le Mystère de la Passion, d'Arnoul Greban / publié d'après les manuscrits de Paris, avec une introduction et un glossaire. Paris: Gallimard, 1987. P. 143. 
ma houlete m'est trop propice;

sans elle n'est riens que je fisse;

encore s'il la desiroit,

je me doubte bien qu'il l'aroit...

- Luy donras tu ton chien?

- Nennin;

qui retourneroit mes brebis?

- Tu luy donras donc du pain bis

et des chastaignes ung grant mont?

- Nennil.

- Que luy donras tu dont?

- Je luy donray mon flageollet... ${ }^{1}$

3. В некоторых случаях вопросительное предложение с SV практически равно утверждению. Говорящий не спрашивает, а скорее с уверенностью утверждает, ожидая от своего собеседника согласия с высказанной точкой зрения.

A) - Il fault donc dire que c'est Crist, promis des prophutes haultains?

- C'est il, nous en sommes certains ${ }^{2}$.

B) Et bon mary, de soy courroucer, qui dist: Vous avez menty, paillarde! Vous l'avez mengée ou caichée quelque part? ${ }^{3}$

B XVI-XVII вв. общий вопрос с SV продолжает использоваться в типичной для него функции - запроса подтверждения информации:

Entre autres un jour il lui dit: Vous estes des champs, mamie? - Voire, Monsieur. - Je m'en doutois bien'.

Oisille dist en riant: "Vous sçavez doncques bien nager?"5

Для структуры SV сохраняется дополнительное значение удивления или негодования:

\footnotetext{
1 Le Mystère de la Passion / d'Arnoul Greban, publié d'après les manuscrits de Paris, avec une introduction et un glossaire. Paris: Gallimard, 1987. P. 70.

2 Le Mystère de la Passion / d'Arnoul Greban, publié d'après les manuscrits de Paris, avec une introduction et un glossaire. Paris: Gallimard, 1987. P. 73.

3 Les Cent Nouvelles nouvelles. Dites les Cent nouvelles du roi Louis XI. Paris: Delahays, 1858. P. 191.

4 Béroalde de Verville F. Le moyen de parvenir. T. 1. Notice, variantes, glossaire et index des noms par Charles Royer, 1896. P. 80.

5 Marguerite de Navarre. L'Heptaméron des nouvelles. T. 2. Paris: Librairie des bibliophiles, 1880. P. 66.
}

Comment, dist Epistemon, tout le monde chevaulchera, et je menerai l'asne? ${ }^{6}$

Вопрос с прямым порядком слов встречается также в вопросах-переспроcax:

- Et si je ne me marie poinct? - Je n'y voi inconvünient aulcun. - Vous n'y en voyez poinct? $-\mathrm{Nul}^{7}$.

Что касается морфосинтаксических особенностей общевопросительного предложения SV-типа в XI-XVII вв., то одним из его характерных признаков является местоимённая природа подлежащего. Как правило, это местоимение 2-го лица, что объясняется преимущественным употреблением вопроса в рамках диалога.

$\underline{\text { Tu }}$ ne sez pas que il t'estuie?

Встречается также местоимение 3-го лица, в составе безличных конструкций:

Il luy fault or? on le luy fourre!

Именное подлежащее также не исключено, хотя и является менее типичным для вопросительного предложения с SV.

- Et ainsi l'enffant a esté

par ce moyen mis en oubly? ${ }^{10}$

Спецификой общих вопросов с прямым порядком слов (которая отличает их от других структурных типов вопроса) является оформление дополнительными лексико-грамматическими элементами, которые подчёркивают разную степень уверенности спрашивающего. Используя данные вставки, говорящий ожидает скорее утвердительного ответа на свой вопрос.

6 Rabelais F. Oeuvres de François Rabelais, contenant la vie de Gargantua et celle de Pantagruel. Paris: Nouvelle Librairie Française, 1957. P. 131.

7 Rabelais F. Oeuvres de François Rabelais, contenant la vie de Gargantua et celle de Pantagruel. Paris: Nouvelle Librairie Francaise, 1957. P. 140.

8 Benoît de Sainte-Maure. Chronique des ducs de Normandie. Vol. II / publ. d'après un manuscrit de musée britannique par Francisque Michel. Paris: Imprimerie Royale, 1838. P. 223

9 La farce de maître Pathelin. Paris: Gallimard, 1999. P. 18.

${ }^{10}$ Le Mystère de la Passion, d'Arnoul Greban / publié d'après les manuscrits de Paris, avec une introduction et un glossaire. Paris: Gallimard, 1987. P. 119. 
Tu es doncques de sa maignie, son disciple, a ce que j'entens?

B XV в. встречается выражение faictes pas?, которое можно считать предшественником современного n'est-ce pas:

Or, Monseigneur, vous avez perdu la gaigeure? Vous le congnoissez bien, faictes pas? Ouy, dist-il, c'est raison'.

\section{Заключение}

Как показало исследование, развитие общего вопроса во французском языке шло по пути от инверсии к прямому порядку слов, однако нельзя сказать, что прямой порядок слов полностью вытеснил инверсию, поскольку в современном языке несколько форм вопроса (SV, est-ce que, инверсия) сосуществуют. В то же время, каждая форма сохраняет свою специфику, которую нужно учитывать.

Согласно проведённому анализу, структура SV занимает промежуточное положение между собственно вопросом и повествовательным предложением. Существует ряд особенностей семантического, синтаксического и фонетического порядка, позволяющих разграничить общевопросительное предложение с прямым порядком слов и простое повествовательное предложение, с одной сторо-

1 Le Mystère de la Passion, d’Arnoul Greban / publié d'après les manuscrits de Paris, avec une introduction et un glossaire. Paris: Gallimard, 1987. P. 227.

2 Les Cent Nouvelles nouvelles. Dites les Cent nouvelles du roi Louis XI. Paris: Delahays, 1858. P. 139. ны, и такие структуры общего вопроса, как инверсия и оборот est-ce que, с другой стороны.

Исследование значительного корпуса фактического материала позволило выявить, что основным значением вопроса c SV является направленность на подтверждение запрашиваемой информации, и, как показывает анализ примеров художественной литературы XI-XVII вв., он используется в более широких контекстах по сравнению с est-ce que и инверсией, приобретая дополнительное значение удивления, недовольства и др.

Общевопросительная конструкция с прямым порядком слов способна сочетаться с дополнительными лексико-грамматическими элементами, усиливающими тот или иной семантический оттенок. Она может функционировать со вставками (donc, alors, sûrement), целью которых является стремление ориентировать, склонить собеседника к какому-либо ответу.

Таким образом, можно заключить, что тенденция увеличения доли структуры SV в вопросительных предложениях объясняется не столько стремлением избежать инверсии, сколько целой совокупностью разнообразных структурносемантических, прагматических и социолингвистических факторов.

Статья поступила в редакицю 12.05.2021

\section{ЛИТЕРАТУРА}

1. Бирюкова И. А. Проблема порядка слов во французском языке в трудах ведущих ученых-лингвистов // Scripta manent. 2015. № 21. С. 6-13.

2. Васильева Н. М. Вопросительное предложение во французском и испанском языках (на французском языке) / La phrase interrogative dans les langues française et espagnole. М.: ЛЕНАНД, 2020. 112 c.

3. Васильева Н. М. Порядок слов в современном французском языке (простое независимое предложение): монография. М.: МГОУ, 2009. 113 с.

4. Еливанова А. М. Порядок слов в предложении во французском языке: диахронический подход // Филологические науки. Вопросы теории и практики. 2013. № 9-1 (27). С. 67-69.

5. Berrendonner A. Questions et mémoire discursive // Les Etats de la question / éd. C. Rossari, A. Beaulieu-Masson, C. Cojocariu, A. Razgoulieva. Québec: Editions Nota Bene, 2005. P. 147-173.

6. Coveney A. L'interrogation directe // Travaux de linguistique. 2011. No. 63. Iss. 2. P. 112-145. DOI : 10.3917/tl.063.0112

7. Delais-Roussarie E., Herment S. Intonation et interprétation des questions: un puzzle pluridimensionnel // L'interrogative en français / éds. M.-J. Béguelin, A. Coveney, A. Guryev. Berne: Peter Lang, 2018. P. 51-79. 
8. Fontaney L. A la lumière de l'intonation // La question / ed. C. Kerbrat-Orecchioni. Lyon : Presses Universitaires de Lyon, 1991. P. 113-161.

9. Foulet L. Comment ont évolué les formes de l'interrogation // Romania. 1921. T. 47. No. 186-187. P. 243-348.

10. Fournier N. Expression et place des constituants dans l'énoncé en français classique: la relation sujetverbe et la relation verbe-objet // Langue française. 2001. No. 130. La linguistique diachronique: grammaticalisation et sémantique du prototype. P. 89-107.

11. Fromaigeat $\mathrm{E}$. Les formes de l'interrogation en français moderne: leur emploi, leurs significations et leur valeur stylistique // Vox romania. 1938. No. 3. P. 1-47.

12. Fuchs C., Le Goffic P. Le français moderne, entre V2 et SVO? // Discours, diachronie, stylistique du français / dir. O. Bertrand, M. Charolles, J. François, S. Prevost, C. Schnedecker. Berne: Peter Lang, 2007. P. $17-35$.

13. Grande Grammaire Historique du Franзais (GGHF) / ed. Ch. Marchello-Nizia, B. Combettes, S. Prevost, T. Scheer: 2 vol. Berlin: De Gruyter Mouton, 2020. 2185 p.

14. Guryev A. Du rôle des paramètres morphosyntaxiques dans la sélection des interrogatives // L'interrogative en français / éds. M.-J. Béguelin, A. Coveney, A. Guryev. Berne: Peter Lang, 2018. P. 153-182.

15. Hansen M.-B. M. Syntax in interaction. Form and function of yes/no interrogatives in spoken standard French // Studies in language. 2001. Vol. 25. Iss. 3. P. 463-520. DOI: 10.1075/sl.25.3.04mos.

16. Marchello-Nizia C. Le français dans l'histoire // Le grand Livre de la langue française / éd. M. Yaguello. Paris: Le Seuil, 2003. P. 11-88.

17. Merle J.-M. La question et l'interrogation en contexte: point de vue énonciatif // Corela (revue en ligne). 2019. HS-29. URL: http://journals.openedition.org/corela/8834 (дата обращения: 24.04.2021). DOI: $10.4000 /$ corela. 8834

18. Muller C. Réflexions sur l'ordre des mots en français (les constituants majeurs de l'énoncé). // Actes du 1er Congrus Mondial de Linguistique Française / ed. J. Durand, B. Habert, B. Laks. Paris: EDP Sciences, 2008. P. 2663-2676

19. Quillard V. La diversité des formes interrogatives - comment l'interpréter? // Langage et société. 2001. No. 95. P. 57-72.

20. Riegel M., Pellat J.-Ch., Rioul R. Grammaire méthodique du français. 7-e éd. Paris: PUF, 2018. 1168 p.

21. Šafárová M. The semantics of rising intonation in interrogatives and declaratives // Proceedings of sinn und bedeutung. 2005. Vol. 9. P. 355-369.

\section{REFERENCES}

1. Biryukova I. A. [The problem of word order in French in the works of leading linguists]. In: Scripta manent, 2015, no. 21, pp. 6-13.

2. Vasil'eva N. M. Voprositel'noe predlozhenie vo frantsuzskom i ispanskom yazykakh (na frantsuzskom yazyke) / La phrase interrogative dans les langues française et espagnole [Interrogative sentence in French and Spanish (in French)]. Moscow, LENAND Publ., 2020. 112 p.

3. Vasil'eva N. M. Poryadok slov v sovremennom frantsuzskom yazyke (prostoe nezavisimoe predlozhenie) [Word order in modern French (simple independent sentence)]. Moscow, MGOU Publ., 2009. 113 p.

4. Elivanova A. M. [Word order in the French language sentence: diachronic approach]. In: Filologicheskie nauki. Voprosy teorii i praktiki [Philology. Theory \& Practice], 2013, no. 9-1 (27), pp. 67-69.

5. Berrendonner A. Questions et mémoire discursive. In: Rossari C., Beaulieu-Masson A., Cojocariu C., Razgoulieva A., éds. Les Etats de la question. Québec, Editions Nota Bene Publ., 2005, pp. 147-173.

6. Coveney A. L'interrogation directe. In: Travaux de linguistique, 2011, no. 63, iss. 2, pp. 112-145. DOI : $10.3917 /$ tl.063.0112

7. Delais-Roussarie E., Herment S. Intonation et interprétation des questions: un puzzle pluridimensionnel. In: Béguelin M.-J., Coveney A., Guryev A., éds. Linterrogative en français. Berne, Peter Lang, 2018, pp. 51-79.

8. Fontaney L. A la lumière de l'intonation. In: Kerbrat-Orecchioni C., ed. La question. Lyon, Presses Universitaires de Lyon, 1991, pp. 113-161.

9. Foulet L. Comment ont évolué les formes de l'interrogation. In : Romania, 1921, T. 47, no. 186-187, pp. 243-348.

10. Fournier N. Expression et place des constituants dans l'énoncé en français classique: la relation sujet-verbe et la relation verbe-objet. In : Langue française, 2001, no. 130, pp. 89-107. 
11. Fromaigeat E. Les formes de l'interrogation en français moderne: leur emploi, leurs significations et leur valeur stylistique. In: Vox romania, 1938, no. 3, pp. 1-47.

12. Fuchs C., Le Goffic P. Le français moderne, entre V2 et SVO? In : Bertrand O., Charolles M., François J., Prevost S., Schnedecker C., dir. Discours, diachronie, stylistique du français. Berne, Peter Lang, 2007, pp. 17-35.

13. Marchello-Nizia Ch., Combettes B., Prevost S., Scheer T., eds. Grande Grammaire Historique du Français (GGHF). Berlin, De Gruyter Mouton, 2020. 2185 p.

14. Guryev A. Du rôle des paramètres morphosyntaxiques dans la sélection des interrogatives. In: Béguelin M.-J., Coveney A., Guryev A., éds. L’interrogative en français. Berne, Peter Lang, 2018, pp. 153-182.

15. Hansen M.-B. M. Syntax in interaction. Form and function of yes/no interrogatives in spoken standard French. In: Studies in language, 2001, vol. 25, iss. 3, pp. 463-520. DOI: 10.1075/sl.25.3.04mos

16. Marchello-Nizia C. Le français dans l'histoire. In: Yaguello M., ed. Le grand Livre de la langue française. Paris, Le Seuil, 2003, pp. 11-88.

17. Merle J.-M. La question et l'interrogation en contexte: point de vue énonciatif. In: Corela (revue en ligne), 2019, HS-29. Available at: http://journals.openedition.org/corela/8834 (accessed: 24.04.2021). DOI: $10.4000 /$ corela. 8834

18. Muller C. Réflexions sur l'ordre des mots en français (les constituants majeurs de l'énoncé). In: Durand J., Habert B., Laks B., eds. Actes du 1er Congrès Mondial de Linguistique Française. Paris, EDP Sciences, 2008, pp. 2663-2676.

19. Quillard V. La diversité des formes interrogatives - comment l'interpréter? In: Langage et société, 2001, no. 95 , pp. 57-72.

20. Riegel M., Pellat J.-Ch., Rioul R. Grammaire méthodique du français. Paris, PUF, 2018. 1168 p.

21. Šafárová M. The semantics of rising intonation in interrogatives and declaratives. In: Proceedings of sinn und bedeutung, 2005, vol. 9, pp. 355-369.

\section{ИНФОРМАЦИЯ ОБ АВТОРАХ}

Зубкова Мария Николаевна - кандидат филологических наук, старший преподаватель кафедры французского языка Московского государственного института международных отношений (университета) Министерства иностранных дел Российской Федерации; e-mail:privezentsevamn@gmail.com;

Дроздова Юлия Андреевна - кандидат филологических наук, доцент кафедры романо-германских языков Всероссийской академии внешней торговли Министерства экономического развития Российской Федерации;

e-mail: connectinfo1@yandex.ru

\section{INFORMATION ABOUT THE AUTHORS}

Maria N. Zubkova - Cand. Sci. (Philology), Senior Lecturer, Department of French Language, Moscow State Institute of International Relations (University) of the Ministry of Foreign Affairs of the Russian Federation;

e-mail: privezentsevamn@gmail.com

Yulia A. Drozdova - Cand. Sci. (Philology), Assoc. Prof., Department of Romance and Germanic languages, Russian Foreign Trade Academy;

e-mail: connectinfo1@yandex.ru

\section{ПРАВИЛЬНАЯ ССЫЛКА НА СТАТЬЮ}

Зубкова М. Н., Дроздова Ю. А. Функционирование общевопросительных предложений с прямым порядком слов во французском языке (на материале художественных текстов XI-XVII веков) // Вестник Московского государственного областного университета. Серия: Лингвистика. 2021. № 5. C. $36-45$.

DOI: $10.18384 / 2310-712 \mathrm{X}-2021-5-36-45$ 


\section{FOR CITATION}

Zubkova M. N., Drozdova Yu. A. Functions of general questions with direct word order in French (based on literary texts of the $11^{\text {th }}-17^{\text {th }}$ centuries). In: Bulletin of the Moscow Region State University. Series: Linguistics, 2021, no. 5, pp. 36-45.

DOI: $10.18384 / 2310-712 \mathrm{X}-2021-5-36-45$ 